\title{
Performance studies of large resistive Micromegas quadruplets in Test-Beams and High Radiation Environments
}

\author{
Ourania Sidiropoulou $* a b$ \\ ${ }^{a}$ CERN EP Department, Geneve ( $\left.\mathrm{CH}\right)$ \\ ${ }^{b}$ Bayerische Julius Max. Universitaet Würzburg (DE) \\ E-mail: ourania.sidiropoulouecern.ch
}

B. Alvarez Gonzalez, ${ }^{a}$ J. Bortfeldt, ${ }^{a}$ A. Düdder, ${ }^{c}$ E. Farina, ${ }^{a d}$ P. lengo, ${ }^{a}$ F. Kuger, ${ }^{a b}$ Tai-Hua Lin, ${ }^{c}$ M. Schott, ${ }^{c}$ G. Sekhniaidze, ${ }^{e}$ F. Sforza, ${ }^{a}$ and J. Wotschack ${ }^{a}$

${ }^{a}$ CERN EP Department, Geneve (CH)

${ }^{b}$ Bayerische Julius Max. Universität Würzburg (DE)

${ }^{c}$ Johannes-Gutenberg-Universität Mainz (DE)

${ }^{d}$ University of Pavia and INFN(IT)

${ }^{e}$ INFN and University of Naples (IT)

\begin{abstract}
In recent years, Micromegas detectors became more and more important for large scale tracking systems in particles physics. The first Micromegas quadruplet that has been built at CERN features an active area of $0.4 \mathrm{~m}^{2}$ per plane and is based on the resistive-strip technology, which can be used for precision tracking in high rate background environments. The detector was built in a modular way. The quadruplet consists of two double-sided readout panels and three support (or drift) panels equipped with the micromesh and the drift electrode. The panels are bolted together such that the detector can be opened and cleaned, if required. Here, we present the results of the panel alignment as measured after assembly of the detector and performance studies in a high-rate radiation environment at the GIF++ facility.
\end{abstract}

38th International Conference on High Energy Physics

3-10 August 2016

Chicago, USA

\footnotetext{
* Speaker.
} 


\section{Introduction}

A resistive Micromegas quadruplet with a mechanically floating mesh and an active area of $0.4 \mathrm{~m}^{2}$ per plane has been built at CERN in 2014, serving as prototype for large scale experiments. The quadruplet consists of 3 drift panels, 2 readout panels with identical micromegas structures on both sides mounted back-to-back, 4 gas gaps forming drift regions of $5 \mathrm{~mm}$ and 2 high-voltage sectors per layer (Figure 1). Details on the construction of this detector are reported in [1]. The micro-mesh (woven stainless steel with $30 \mu \mathrm{m}$ diameter wires and $50 \mu \mathrm{m}$ opening) is supported by the drift panel in order to facilitate detector opening and cleaning. When the detector is closed the mesh is resting on pillars attached to the readout board and defining an amplification gap of $128 \mu \mathrm{m}$ (pillar height). Each readout layer comprises 1024 copper readout strips with $415 \mu \mathrm{m}$ pitch and a width of $315 \mu \mathrm{m}$. The readout strips are covered by a $50 \mu \mathrm{m}$ thick Kapton foil carrying resistive strips of approximately $1 \mathrm{M} \Omega / \mathrm{sq}$ to limit discharge currents. In one readout panel the strips are parallel to the base of the trapezoid in order to measure the precision coordinate $(\eta)$ while in the other panel they are rotated by $\pm 1.5^{\circ}$ to measure the hit position in the precision and the transverse coordinate $(\phi)$. In this paper we present the mechanical accuracy measured after assembly as well as the detector performance under intense photon irradiation. The detector is operated with an Ar: $\mathrm{CO}_{2}$ 93:7 vol. \% mixture at atmospheric pressure. The mesh is grounded, the resistive strips are connected to positive high-voltage and the drift electrode is connected to negative high-voltage.
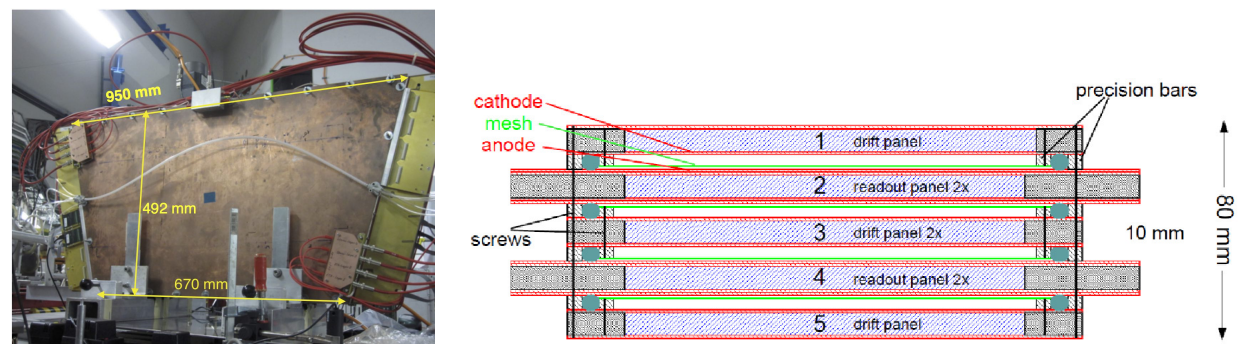

Figure 1: The Micromegas quadruplet prototype (left) and the layout of the detector (right); it consists of three drift and two readout panels.

\section{Strip Alignment}

The alignment of the readout layers is of prime importance for the reconstruction of tracks. The goal was to know the relative position of the strips to better than $40 \mu \mathrm{m}$. The relative alignment of the two $\eta$ readout layers was measured before assembly to be better than $20 \mu \mathrm{m}$ as reported in [1]. After assembly the alignment was measured using an Amptek®Mini-X Silver X-ray source by irradiating the detector surface and recording the charge and time information with the APV-25 Front End ASICs [2] ${ }^{1}$. The X-ray source had a collimator of $2 \mathrm{~mm}$ and was shielded in a copper box. In order to compensate for a possible inclination of the gun inside the box, different runs were

\footnotetext{
${ }^{1}$ The APV-25 Front End ASICs were connected to the ADC and Front-End Concentrator Card (FEC) [3] of the RD51 Scalable Readout System (SRS) and the gigabit Ethernet output was connected to a data acquisition (DAQ-PC) computer running a dedicated DAQ software
} 
taken by rotating the box at angles $0^{\circ}$ and $180^{\circ}$. Figure 2 shows the photon beam profile in one of the readout layers. The profile was fit with a double Gaussian in order to extract the mean value for each angle. The average value between the two angles was calculated for the same readout layer and then the difference between the beam position on the two layers was extracted giving a relative alignment of better than $20 \mu \mathrm{m}$ (Table 1).

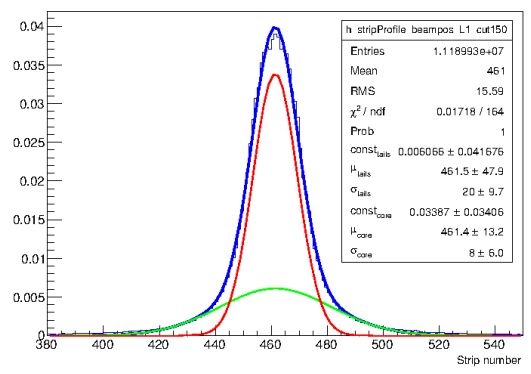

Figure 2: Intensity profile in one readout layer, irradiated with the Amptek Mini-X Silver source with a collimator of $2 \mathrm{~mm}$.

Table 1: Misalignment of the $\eta$ readout panel

\begin{tabular}{|c|c|c|c|c|}
\hline Angle & Layer & Mean Gaus Core [strips] & Mean [strips] & Diff (L1-L2) [ $\mu \mathrm{m}]$ \\
\hline 0 & 1 & 461.41 & \multirow{2}{*}{463.04} & \multirow{5}{*}{17} \\
\hline 180 & 1 & 464.67 & & \\
\hline & & & & \\
\hline 0 & 2 & 460.29 & \multirow{2}{*}{463.0} & \\
\hline 180 & 2 & 465.70 & & \\
\hline
\end{tabular}

\section{Studies at the CERN Gamma Irradiation Facility (GIF++)}

\subsection{The GIF++ Facility}

The new GIF++ facility is located in the North Area of the CERN Super Proton Synchrotron. It features a ${ }^{137} \mathrm{Cs}$ source with an activity of about $14 \mathrm{TBq}$ and a half-life of about 30 years. The high gamma flux can be combined with high energetic muons, giving the possibility to study the detector performance in a photon background. The gamma ray flux can be adjusted by a system of filters.

\subsection{Ageing Studies}

In summer 2016 the quadruplet prototype was placed in GIF++ in order to study its performance. Figure 3 shows the integrated charge for all layers after one month of irradiation. The accumulated charge of $2 \mathrm{mC} / \mathrm{cm}^{2}$ is explained by the reduced duty cycle of the source during test beam measurements. We are aiming at accumulating more than $0.2 \mathrm{C} / \mathrm{cm}^{2}$, which is the maximum equivalent charge expected after 10 years of High Luminosity LHC operation in the ATLAS New Small Wheel region [4]. This will be reached after approximately 2 years of irradiation in GIF++. 


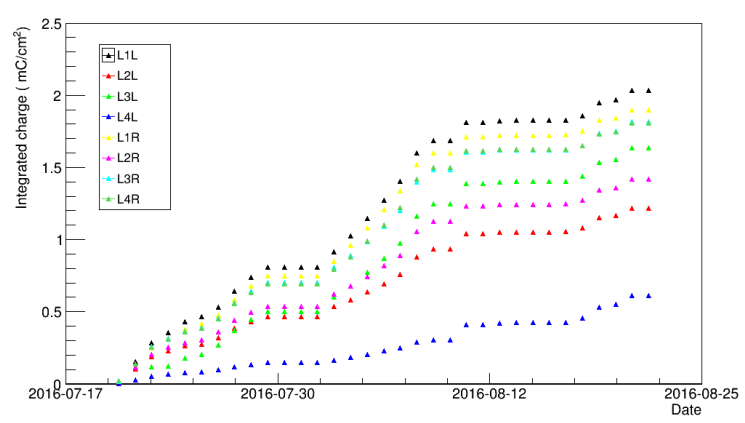

Figure 3: Integrated charge in one month of irradiation in the Micromegas quadruplet. Each readout layer is separated in two high-voltage sectors, right and left. The corresponding high-voltage sectors are denoted as "R" and "L" respectively.

\subsection{Tracking with Muon Beam}

The performance of the quadruplet prototype has been studied by exposing the detector to a high-energy muon beam in the presence of high-rate photon background.

Figure 4 shows the experimental set-up. Four Micromegas detectors with an active area of $9 \times 9 \mathrm{~cm}^{2}$ were used to reconstruct muon tracks. They are located up- and downstream of the quadruplet (MMSW). Three of them have two readout layers with $250 \mu \mathrm{m}$ pitch in $\mathrm{X}$ and perpendicular Y direction (MM1, MM2, MM3), while the fourth features one readout layer with a strip pitch of $400 \mu \mathrm{m}$ (TLP).

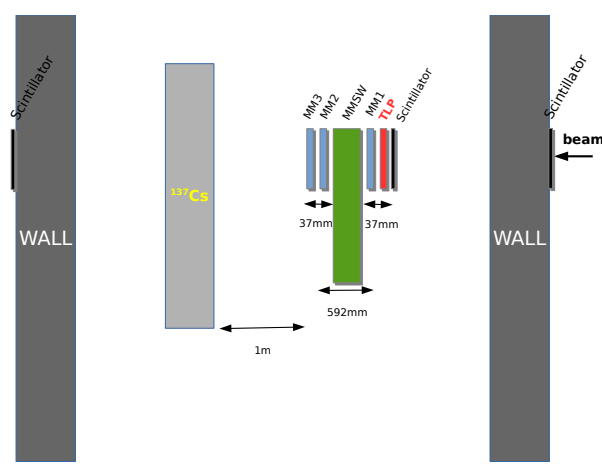

Figure 4: Experimental set-up at GIF++, not to scale.

Figure 5 (left) shows the distribution of the pulse height for the two $\eta$ layers of the MMSW without background irradiation. This distribution is fitted with a Landau distribution and the most probable value (MPV) is shown as a function of the amplification field on the right. The pulse height (which is proportional to the gas gain) increases exponential as expected. The difference between L1 and L2 is (probably) explained by a large gas leak in the O-ring of Gap 2. To compensate for this L2 was operated at $10 \mathrm{~V}$ higher than L1.

The spatial resolution of the two $\eta$ layers without irradiation is shown in Figure 6 as a function of the drift and the amplification field. Here the resolution ${ }^{2}$ is defined as the difference between the

\footnotetext{
${ }^{2}$ The spatial resolution of the two readout layers should be identical thus the hit position difference can be divided by $\sqrt{2}$.
} 

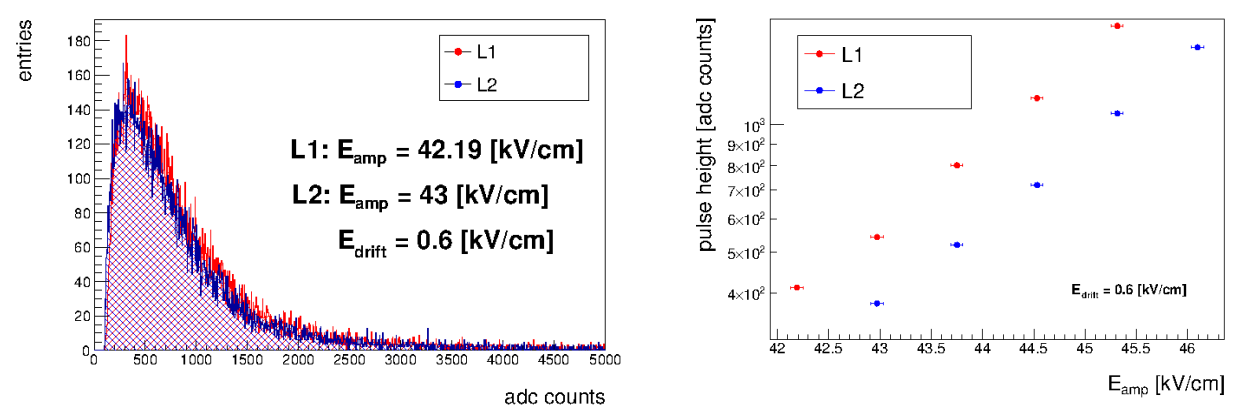

Figure 5: Pulse height distribution of the two $\eta$ layers without background irradiation at low amplification field (left) and the MPV as a function of the amplification field without background irradiation (right)

hit position on the two layers divided by $\sqrt{2}$.
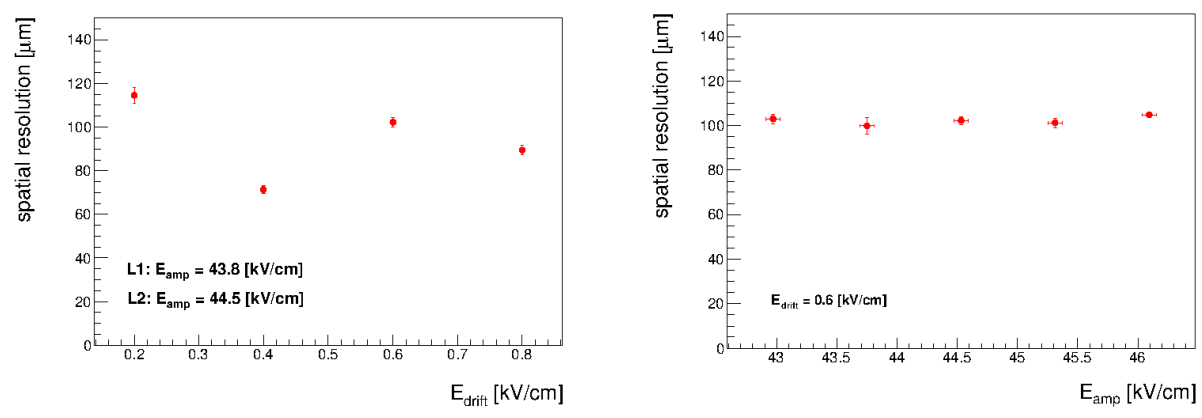

Figure 6: Spatial resolution as a function of the drift (left) and the amplification field (right) without irradiation.

During irradiation in GIF++ muon tracks are distinguished from photons using a Hough transform based algorithm[5]. The residual of the measured hit in a Micromegas layer with respect to the hit predicted by the reference detectors is shown in Figure 7 (left) as a function of the amplification field with and without irradiation. A source attenuation of 10 corresponds to a hit rate of $18 \mathrm{kHz} / \mathrm{cm}^{2}$. The uncertainty of the track was subtracted from the calculation of the spatial resolution. The difference in the resolution with and without irradiation is due to the pattern recognition that is not optimized for background hits. The pulse height behaviour shown in Figure 7 (right) with and without irradiation reveals that the detector is working as expected.

\subsection{Conclusions}

A resistive Micromegas quadruplet prototype with mechanically floating mesh and an active area of $0.4 \mathrm{~m}^{2}$ per layer was built at CERN. The relative alignment of the two $\eta$ readout layers was measured before and after assembly of the detector with different techniques. In both measurements the misalignment was measured to be less than $20 \mu \mathrm{m}$. The detector was placed in the Gamma 

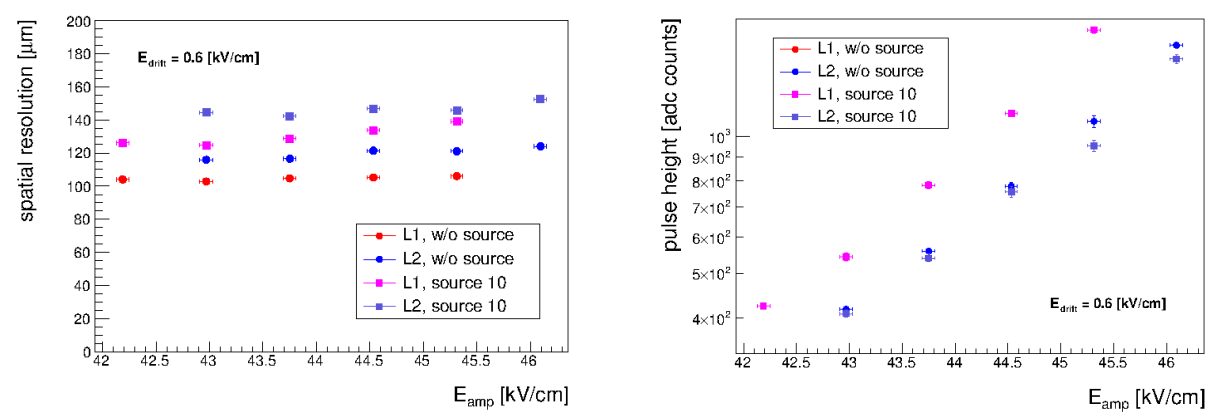

Figure 7: Spatial resolution (left) and pulse height (right) as a function of the amplification field with and without irradiation for the two $\eta$ layers. In the right Fig. the data points for L1 without source are hidden by the points for source attenuation 10 .

Irradiation Facility at CERN for ageing studies. The performance of the detector was studied during a test beam period. The response of the detector with and without irradiation is equal and as expected. The spatial resolution with irradiation, likely caused by the non optimised track and hit reconstruction algorithm, was slightly worse for data with background. It was found after the conference that the system suffered from gas leaks and thus the MMSW detector has not been operated under optimal conditions. Therefore, the performance results should be considered as preliminary, final results are expected to be better.

\section{Acknowledgments}

O.Sidiropoulou was supported by the Wolfgang Gentner Program of the Federal Ministry of Education and Research: http://cern.ch/gentner and E. Farina by the H2020 project AIDA-2020, GA no. 654168.

\section{References}

[1] M. Bianco et al., Nucl. Instr. Meth. A 814 (2016) 117-130

[2] M. Raymond et al., IEEE Nucl. Sci. Symp. Conf. Rec. 2 (2000) 9/113

[3] S. Martoiu et al., JINST 8 (2013) $\mathbf{C 0 3 0 1 5}$

[4] ATLAS Collaboration, CERN-LHCC-97-022

[5] P.V.C. Hough Conf. Proc. C590914 (1959) 554 